\title{
Anesthetic experience using total intra-venous anesthesia for a patient with Wolf-Hirschhorn syndrome -A case report-
}

\author{
Jae Ho Choi, Jae Hwan Kim, Young Cheol Park, Woon Young Kim, and Yoon-Sook Lee \\ Department of Anesthesiology and Pain Medicine, Korea University Ansan Hospital, Ansan, Korea
}

We present here the case of a 33-month-old male patient with Wolf-Hirschhorn syndrome (WHS) and who underwent tympanoplasty and myringotomy. WHS is caused by a rare chromosomal abnormality, which is the deletion of the short arm of chromosome number 4. The typical craniofacial features of WHS patients such as micrognathia, microcephaly and the muscular weakness can make using neuromuscular blocking agents and performing intubation difficult. Moreover, there are a few previous case reports showing that malignant hyperthermia occurred during and after an operation in which the anesthesia was done with inhalation agents, so special anesthetic care is needed when operating on a WHS patient. By carefully intubating the patient and using total intravenous anesthesia, we performed successful anesthesia without any complications. We describe here the anesthetic management of a WHS patient and we review the relevant literature. (Korean J Anesthesiol 2011; 60: 119-123)

Key Words: Difficult intubation, Malignant hyperthermia, Total intra-venous anesthesia, Wolf-hirschhorn syndrome.

Wolf-Hirschhorn syndrome (WHS) is a very rare hereditary disease that shows a deletion of the distal short arm of chromosome 4 and particularly the 4p16.3 domain [1]. Patients with WHS are characterized by typical craniofacial features such as hypertelorism and a "Greek warrior helmet appearance" of the nose, microcephaly, a high forehead with prominent glabella, ocular hypertelorism, epicanthus, highly arched eyebrows, a short philtrum, a down-turned mouth, micrognathia and underdeveloped ears $[1,2]$. The prevalence of WHS has been reported to be approximately $1: 50,000$ and it shows a female predilection at a ratio of $2: 1$ [1]. All these patients have prenatal-onset growth deficiency, postnatal growth retardation and hypotonia with under-developed muscles [2]. These patients have characteristic musculo-skeletal anomalies, congenital heart defects, hearing loss, urinary tract malformations and structural brain abnormalities. Patients with WHS commonly have the concurrent presence of seizure, and the prognosis of these patients is very poor. Thirty-four percent of these patients die of cardiac failure or pneumonia at the age of $<2$ years [1-3].

Received: April 3, 2010. Revised: September 13, 2010. Accepted: October 4, 2010.

Corresponding author: Jae Hwan Kim, M.D., Ph.D., Department of Anesthesiology and Pain Medicine, Korea University Ansan Hospital, 516, Gojan 1- dong, Danwon-gu, Ansan 425-707, Korea. Tel: 82-31-412-5295, Fax: 82-31-412-5294, E-mail: anejhkim@korea.ac.kr

(c) This is an open-access article distributed under the terms of the Creative Commons Attribution Non-Commercial License (http:// creativecommons.org/licenses/by-nc/3.0/), which permits unrestricted non-commercial use, distribution, and reproduction in any medium, provided the original work is properly cited. 
A diagnosis of WHS is tentatively made based on the findings such as the gross facial appearance, developmental delay, psychomotor retardation and seizure, and it is established based on a loss of the Wolf-Hirschhorn critical region (WHCR) (chromosome 4p16). Using fluorescence in situ hybridization, a loss of the WHCR can be confirmed at a frequency of $>95 \%[1,2]$.

Patients with WHS are in need of corrective surgery due to the presence of various types of anomalies, and general anesthesia is performed in many cases. But due to the presence of various types of anomalies, managing the airway, regulating the muscle relaxation and controlling the vital signs during the surgery can be difficult. Moreover, there are also case studies that have reported the postoperative occurrence of malignant hyperthermia [4,5]. We performed total intravenous anesthesia (TIVA) in a patient who was diagnosed with WHS. We report here on our case along with a review of the relevant literature.

\section{Case Report}

A 33-month-old male baby whose height and weight were $88 \mathrm{~cm}$ and $11.35 \mathrm{~kg}$, respectively, visited our hospital to undergo tympanoplasty and myringotomy for the bilateral ears under general anesthesia. The patient was born through a normal delivery and he had a weight of $2.67 \mathrm{~kg}$ at birth. From approximately one year old on, the patient presented several times with febrile seizure. At the time of admission, the patient had a height and a weight that were less than the

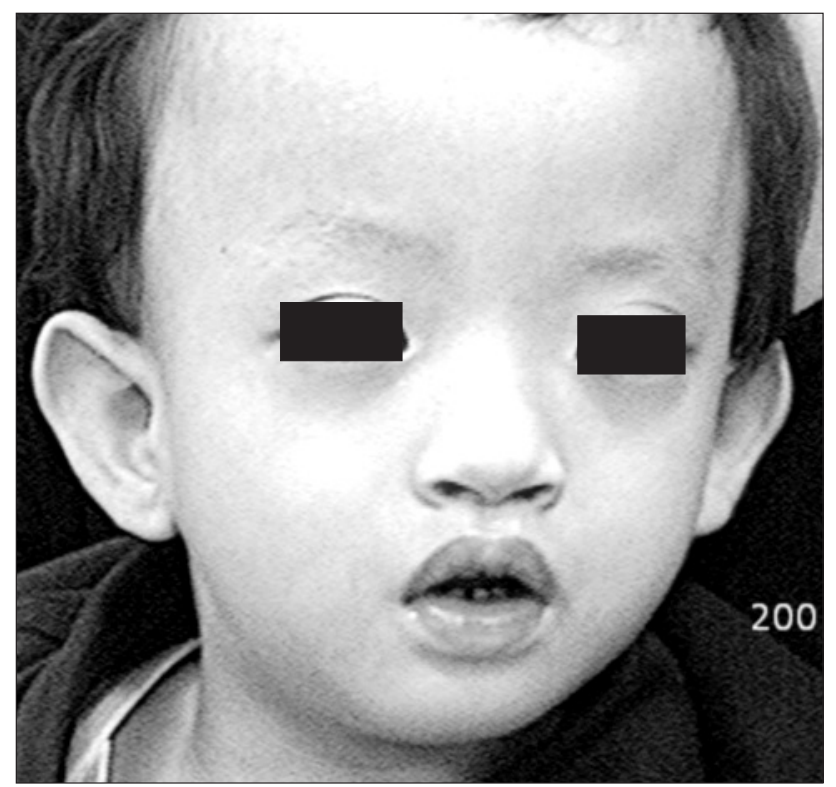

Fig. 1. The patient has typical craniofacial features such as microcephaly, a high forehead with prominent glabella, ocular hypertelorism, epicanthus, highly arched eyebrows, a short philtrum, a down-turned mouth and micrognathia. third percentile. The patient's motor ability was such that he could not stand alone without manual support. The patient also presented with such symptoms as motor and intellectual developmental delay and he had no responses to toys.

The patient also had microcephaly and characteristic facial dysmorphism (Fig. 1). Both parents had no abnormal findings in their family medical histories. The patient had never experienced anesthesia. The parents had normal findings on genetic testing. But on gene analysis, the patient had $46 X Y \operatorname{der}(4) \mathrm{t}(4 ; ;)(\mathrm{p} 16 ; ;)$ and this led to the diagnosis of WHS (Fig. 2). Due to continual rehabilitation, the patient could respond to calling his name. The patient also had an attention span of approximately 20 minutes in length and the verbal communication ability of approximately an 11 month old child. The patient frequently fell due to his unstable gait, but his developmental status was such that he could walk alone.

On physical examination, the patient had no other findings other than what had been previously confirmed. There were no notable findings on the pre-operative work-up. There were no other prescribed drugs except phenobarbital for the management of seizure. The patient showed normal cardiac rhythm on echocardiography (EKG) and on auscultation, the patient showed normal cardiac sounds without a cardiac murmur. On the airway test, the patient had an inter-incisional distance of $3.1 \mathrm{~cm}$ for the maximal mouth opening and a thyromental distance of $4.3 \mathrm{~cm}$, which extended from the thyroid notch to the chin. Based on these findings, the patient was grade II on the classification system of Mallampati. The neck movement showed flexion of $>35$ degrees and extension of $>80$ degrees. These results showed that there were no restrictions of neck movement. Additionally, to confirm the presence of any anomaly of the airway tract in the patient, anterior and posterior radiography was done together with

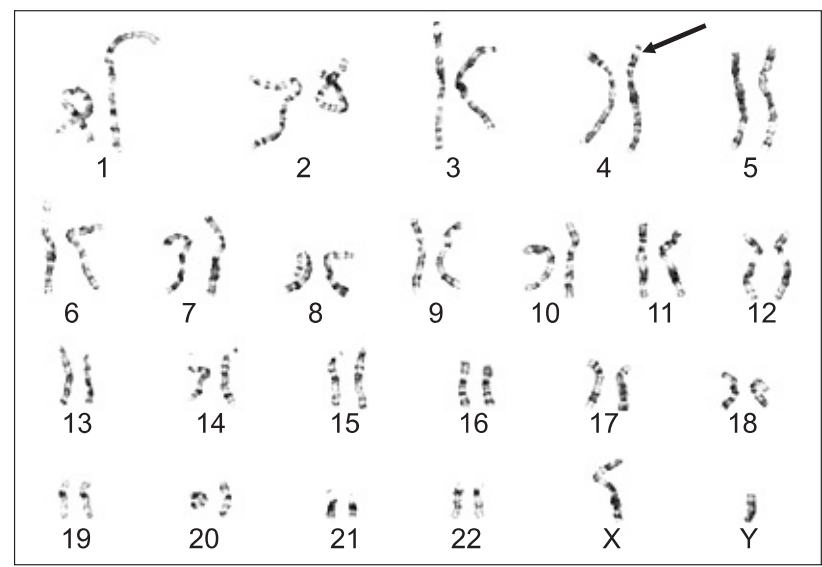

Fig. 2. Chromosomal study: $46 \mathrm{XY} \operatorname{der}(4) \mathrm{t}(4 ; ;)(\mathrm{p} 16 ; ;)$. There is a deletion of genetic material near the end of the short (p16) arm of chromosome 4. 
lateral radiography of the cervical spine, but there were no abnormal findings.

The patient was given an intramuscular injection of atropine $0.2 \mathrm{mg}$ prior to anesthesia. The preoperative vital signs included a heart rate of 110 times/min, a blood pressure of 90/60 $\mathrm{mmHg}$, a respiratory rate of 20 breaths/min and a body temperature of $36.2^{\circ} \mathrm{C}$. For the intraoperative management, the end-tidal partial pressure of carbon dioxide, the body temperature via a nasal cavity and the oxygen saturation were measured, along with monitoring the EKG. Prior to the induction of anesthesia, due to the concern for the difficulty of endotracheal intubation, oxygen was provided to a sufficient extent for more than approximately three minutes. For the induction and maintenance of anesthesia, midazolam $0.1 \mathrm{mg} / \mathrm{kg}$ was intravenously injected once by using a remifentanil continuous infusion system (Orchestra $^{\circledR}$, Base Primea, Fresinius Vial, France), and this was set at a rate of $0.20-0.40 \mu \mathrm{g} / \mathrm{kg} / \mathrm{min}$. Following confirmation of easy ventilation, rocuronium $0.6 \mathrm{mg} / \mathrm{kg}$ was intravenously injected. Respiratory control was done using a mask. Endotracheal intubation was performed using a tube with an intratracheal lumen of $4.0 \mathrm{~mm}$, which is a smaller size than is normally used for a patient of this age. For the laryngoscopic view, the patient was grade IIa on the modified Cormack and Lehane scoring system. Only part of patient's glottis and vocal cords were visible, but endotracheal intubation was completed with no great difficulty. Oxygen and air were supplied at a rate of $2 \mathrm{~L} / \mathrm{min}$ and $3 \mathrm{~L} / \mathrm{min}$, respectively. The intraoperative vital signs included a heart rate of 150-170 times/min and oxygen saturation of $97-100 \%$. During anesthesia, the patient showed no specific abnormalities of the vital signs, and midazolam 0.1 $\mathrm{mg} / \mathrm{kg}$ was further administered during surgery to maintain the anesthesia. The surgical time was 25 minutes and the anesthetic time was 40 minutes. The body temperature was $37.4^{\circ} \mathrm{C}$ and $37.9^{\circ} \mathrm{C}$ immediately after surgery was initiated and immediately before it was completed, respectively. The end-tidal partial pressure of carbon dioxide was maintained at $30-35 \mathrm{mmHg}$. The surgical procedure was completed with no great difficulty. After the patient made a recovery of spontaneous respiration to a sufficient extent, glycopyrrolate $0.1 \mathrm{mg}$ and pyridostigmine 2.5 mg were intravenously injected to reverse the muscle relaxation, and this was followed by extubation. The time from the end of surgery to extubation was about 10 minutes. A total of $25 \mathrm{ml}$ of fluids, including $1: 4$ dextrose and sodium chloride solution, had been intraoperatively administered. The patient showed stable vital signs without notable complications in a recovery room. Due to the identification of a fever of $37.8^{\circ} \mathrm{C}$, the patient received continual measurement of the body temperature.

The fever persisted following discharge from the recovery room. Seven hours following the discharge from a recovery room, the patient had a high fever of $39.0^{\circ} \mathrm{C}$ and he had a seizure.
The seizure was controlled by intravenous phenobarbital. But the high fever was persistently present even following the administration of antipyretics and antiepileptics. This led to the suspicion of delayed malignant hyperthermia. Yet the patient showed a congested and enlarged laryngopharynx following consultation with a pediatrician and an otolaryngologist. These findings were suggestive of an upper respiratory infection, so we administered antibiotics and additional antipyretics. The body temperature was normalized two days following the discharge from the recovery room. The patient was assumed to have had a fever due to upper respiratory infection rather than malignant hyperthermia. Thereafter, the patient recovered with no specific symptoms. The patient was discharged from the hospital on the sixth postoperative day.

\section{Discussion}

According to Ginsburg and Purcell-Jones [5], malignant hyperthermia occurred in a 21-month-old female baby with WHS while anesthesia was being maintained with halothane for repairing a cleft palate. Those authors noted that good treatment outcomes were obtained using temperature-lowering therapy and dantrolene infusion. That study was the first reported case in which malignant hyperthermia occurred in a patient with WHS. According to Chen et al. [4], following the induction of anesthesia using halothane, succinylcholine and atracurium, malignant hyperthermia occurred two hours after a surgical procedure was initiated in a WHS patient. That was the first reported case in which the delayed type of malignant hyperthermia occurred in a WHS patient even following surgery. It has also been reported that the delayed type of malignant hyperthermia can occur even 11 hours after the completion of a surgical procedure [6].

In our current study, a delayed type of malignant hyperthermia was suspected seven hours after the child was discharged from a recovery room due to the presence of a high fever of 39.0 and this high was accompanied by seizure. The symptoms of the delayed type of malignant hyperthermia are tachypnea, tachycardia, hyperthermia and metabolic acidosis etc. [6]. But our patient showed all these symptoms of the delayed type of malignant hyperthermia except metabolic acidosis. So it was difficult to diagnose the disease. If such symptoms are developed, anesthesiologists must consider other possible diseases such as malignant hyperthermia, atelectasis, infections, overheating, a reaction to medications and so on [7]. But the patient in our case showed a congested and enlarged laryngopharynx and these findings were suggestive of an upper respiratory infection. Further, the symptoms disappeared after administering antibiotics and additional antipyretics. So we think that the fever developed due to an upper respiratory 
infection rather than being due to the delayed type of malignant hyperthermia.

During induction and maintenance of anesthesia for a patient with WHS, anesthesiologist must not use any drugs and perform procedures that could trigger malignant hyperthermia [7]. The anesthetic drugs that trigger malignant hyperthermia include ether, halothane, enflurane, isoflurane, sevoflurane, and the only currently used depolarizing muscle relaxants (succinylcholine) [8]. The safe anesthetics consist of nitrous oxide, xenon, barbiturates, benzodiazepines, etomidate, propofol, narcotics, local anesthetics and nondepolarizing muscle relaxants $[7,8]$. Before using anesthetic machines for patients who are susceptible to malignant hyperthermia, the anesthetic machines need to be cleansed of potent volatile agents by removal or sealing of the vaporizers, changing the soda lime, perhaps replacing a fresh gas outlet hose, and using a disposable circuit with a flow of $10 \mathrm{~L} / \mathrm{min}$ for 5 minutes $[7,8]$.

If fever develops in the patient with WHS during and after anesthesia, then anestheologists should consider the possibilities of malignant hyperthermia and other causes too. A variety of unusual conditions may resemble malignant hyperthermia during anesthesia. These include iatrogenic overheating, infection, transfusion reaction, central nervous system dysfunction, allergic reactions, pheochromocytoma, thyrotoxicosis, drug induced hyperthermia (i.e., tricyclic antidepressants, monoamine oxidase inhibitors, anticholinergics, amphetamines etc.), machine valve malfunction and rebreathing, as well as faulty equipment [7-9]. The presence of an elevated temperature in the immediate postoperative patient can be associated with conditions such as sepsis or a reaction to medication and this requires appropriate investigation [7]. The response to antipyretics as well the clinical setting is often helpful in differentiating this response from malignant hyperthermia.

According to Ginsburg and Purcell-Jones [5], during the induction of 21-month-old female baby with WHS, tracheal intubation was difficult because of poor visualization of the glottis and intubation was eventually achieved with $3.5 \mathrm{~mm}$ endotracheal intubation tube, which is smaller than usual. Special attention should be paid to the airway management because patients with WHS have various craniofacial anomalies. Before the induction of anesthesia, the patient's airway tract must be intensively examined and anestheologists should prepare for any possibility of difficult intubation [10].

After a patient's airway tract is thoroughly examined, induction of anesthesia in patients with craniofacial anomalies should be carefully done $[10,11]$. Maintaining spontaneous breathing and the face mask ventilability are very important. Premedication agents that do not suppress spontaneous ventilation or the airway reflexes should be used [11]. Either traditional preoxygenation ( 3 or more minutes of tidal volume ventilation) or fast-track preoxygenation (i.e., four maximal breaths in $30 \mathrm{~s}$ ) is effective in delaying arterial desaturation during subsequent apnea [10]. If face mask ventilability is confirmed, then a deeper sedation level or general anesthesia may be performed so as to ease performing laryngoscopy or intubation. But if ventilation is difficult or impossible, then neuromuscular blocking agent should be avoided [11]. To confront a critical situation, it is considered mandatory that all the necessary instruments and devices should be easily and readily available $[10,11]$. We thought that endotracheal intubation could be difficult in our patient, so we carefully took the patient's medical history, we examined the airway tract and the relevant tests were performed. There were no typical abnormalities observed. We conducted all the procedures with caution, so endotracheal intubation was done with no great difficulty and the induction of anesthesia could be safely done.

We report on our anesthetic experience with a WHS patient who had the typical craniofacial features, anomalies of multiple organs and developmental delay as a result of the deletion of p16.3 of chromosome 4. Difficult endotracheal intubation and the occurrence of malignant hyperthermia can be predicted when performing anesthesia for patients with WHS. With careful anesthetic managements, we were able to perform successful, safe anesthesia. However, it should be kept in mind that a high fever after anesthesia in WHS patient could develop due to not only malignant hyperthermia but also other causes such as infection, under-lying disease, iatrogenic causes, equipment problems and so on.

\section{References}

1. Battaglia A, Filippi T, Carey JC. Update on the clinical features and natural history of Wolf-Hirschhorn (4p-) syndrome: experience with 87 patients and recommendations for routine health supervision. Am J Med Genet C Semin Med Genet 2008; 148C: 246-51.

2. Battaglia A, Carey JC, South ST, Wright TJ. Wolf-Hirschhorn Syndrome. In: GeneReviews [PMID: 20301362 ]. Edited by Pagon RA, Bird TD, Dolan CR, Stephens K: Seattle (WA), University of Washington, Seattle. 2002 Apr [updated 2010 Jun]. Available from http://www.ncbi.nlm.nih.gov/bookshelf/ br.fcgi?book=gene\&part=whs.

3. Shannon NL, Maltby EL, Rigby AS, Quarrell OW. An epidemiological study of Wolf-Hirschhorn syndrome: life expectancy and cause of mortality. J Med Genet 2001; 38: 674-9.

4. Chen JC, Jen RK, Hsu YW, Ke YB, Hwang JJ, Wu KH, et al. 4Psyndrome (Wolf-Hirschhorn syndrome) complicated with delay onset of malignant hyperthermia: a case report. Acta Anaesthesiol Sin 1994; 32: 275-8.

5. Ginsburg R, Purcell-Jones G. Malignant hyperthermia in the WolfHirschhorn syndrome. Anaesthesia 1988; 43: 386-8.

6. Souliere CR Jr, Weintraub SJ, Kirchner JC. Markedly delayed 
postoperative malignant hyperthermia. Arch Otolaryngol Head Neck Surg 1986; 112: 564-6.

7. Muldoon S, Sambuughin N, Voelkel M, Bunger R, Grocott HP, Sulzer C. Malignant hyperthermia, thermoregulation, and perioperative hypothermia. In: Anesthesiology. Edited by Longnecker DE, Brown DL, Newman MF, Zapol WM: The United States, The McGraw-Hill companies. 2008, pp 1964-98.

8. Gerald AG, Isaac NP, Heila MM, Timothy JT. Malignant Hyperthermia. In: Miller's Anesthesia. 6th ed. Edited by Miller RD: Philadelphia, Elsevier Churchill Livingstone. 2005, pp 1169-90.

9. Rosenberg H, Davis M, James D, Pollock N, Stowell K. Malignant hyperthermia. Orphanet J Rare Dis 2007; 2: 21.

10. American Society of Anesthesiologists Task Force on Management of the Difficult Airway. Practice guidelines for management of the difficult airway: an updated report by the American Society of Anesthesiologists Task Force on Management of the Difficult Airway. Anesthesiology 2003; 98: 1269-77.

11. Gruppo di Studio SIAARTI "Vie Aeree Difficili", Frova G, Guarino A, Petrini F, Merli G, Sorbello M, et al. Recommendations for airway control and difficult airway management in paediatric patients. Minerva Anestesiol 2006; 72: 723-48. 